\title{
Investigating the route to flutter in a pitch-plunge airfoil with freeplay nonlinearity subjected to input flow fluctuations
}

\author{
Magu Raam Prasaad $\mathrm{R}^{1}$ and Venkatramani $\mathrm{J}^{*}, 1$ \\ ${ }^{1}$ Shiv Nadar University, Mechanical Department, Gautam Buddha Nagar, Uttar Pradesh 201314, \\ India
}

\begin{abstract}
Aeroelastic systems with freeplay nonlinearity can exhibit a wide variety of qualitatively different dynamical responses such as limit cycle oscillations and chaos in the pre-flutter regimes. Consequently, the bifurcation scenario in an aeroelastic system with freeplay nonlinearity under uniform flows have received considerable attention in the literature. However, in reality flows are far from deterministic and often possess a small temporal random fluctuations about a mean value. Input flow fluctuations have the potential to alter the stability and give rise to atypical routes to flutter. Indeed, recent studies have shown that under flow fluctuations the aeroelastic systems loses its stability via a regime of oscillations called intermittency. Further, it is observed that the presence of cubic hardening nonlinearity and input flow fluctuations with predominantly long time scales can give rise to "on-off" type intermittency. This dynamical behaviour is attributed to type of nonlinearity and relatively short time scale for the system to stay and exhibit distinct dynamics. Extending the mechanism of intermittency route to flutter in aeroelastic systems with other prominent types of nonlinearities, such as, freeplay have however, received minimal attention in the literature. The present study devotes itself to investigate the response dynamics of an airfoil with freeplay nonlinearity subjected to long time scale input flow fluctuations.
\end{abstract}

\section{Introduction}

The topic of aeroelasticity is a multidisciplinary study focussing on the interplay between fluid, structural and inertial forces. The mutual interaction between aerodynamic forces and the structural motion often results in harmful aeroelastic oscillations called flutter [1]. This dynamical instability arises when the incoming flow speed is greater than a critical value of flow speed. In such a situation, the airfoil displays violent oscillations that can either cause abrupt structural failure or an accumulation of fatigue damage. Estimating the onset of these detrimental flutter oscillations therefore is an important step in the design of aerospace structures. Identification of flutter regimes, especially, in the ubiquitous presence of non-

\footnotetext{
* Corresponding author: j.venkatramani@snu.edu.in
} 
smooth nonlinearity (such as freeplay) is not a trivial task [2]. Indeed, in the presence of freeplay nonlinearity, it has been observed that nonlinear responses such as limit cycle oscillations (LCOs), period doubling and chaos [3,4]. Consequently, one resorts to performing a bifurcation analysis to investigate the stability characteristics of such nonlinear systems.

Another difficulty that arises in identifying unstable oscillatory regimes is the randomly fluctuating nature of the input wind. These fluctuations alter the stability regimes $[5,6]$ and also give rise to atypical routes to flutter $[7,6]$. Despite the necessity to incorporate flow fluctuations, studies on bifurcation characteristics of freeplay aeroelastic systems have by and large devoted minimal attention to the same. The present study addresses this concern. This study devotes itself to investigate the response dynamics of an airfoil with free play nonlinearity subjected to long time scale input flow fluctuations. A pitch-plunge airfoil with a freeplay nonlinearity in the pitch degree of freedom is considered. A linear aerodynamical formulation accounting for the unsteady wake effects via Wagner function formulation is considered. The fluctuating flow is modelled using a representative sinusoidal term with frequency terms varying randomly with time. With the mean flow speed as the bifurcation parameter, a response analysis is carried out and the route(s) to flutter are identified. Subsequently, a stochastic bifurcation analysis is undertaken to characterize these pre-flutter responses in both qualitative and quantitative paradigms.

The organization of this paper is as follows. Section 2 describes the mathematical model of the airfoil and the flow fluctuations. The results obtained are discussed in Section 3. The salient conclusions that emerge from this study are summarized in Section 4.

\section{Mathematical model}

The airfoil is modelled as a "typical section" - implying a rigid flat plate consisting of two degrees of freedom, namely, pitch and plunge. Flexibility in these degrees of freedom are incorporated by attaching translational and torsional springs at the elastic axis. It is assumed that the pitch possesses a non-smooth form of nonlinearity called freeplay and is modelled as a bilinear stiffness. The incoming flow is considered to be linear and attached. The resulting aerodynamic forces are modelled with a dependency on both the airfoil motion and the wake effects via an unsteady aerodynamic formulation. The resulting governing equations of motion are as follows:

$$
\begin{aligned}
& \epsilon^{\prime \prime}+x_{\alpha} \alpha^{\prime \prime}+2 \zeta_{\epsilon} \frac{\bar{\omega}}{U} \epsilon^{\prime}+\left(\frac{\bar{\omega}}{U}\right)^{2} \epsilon=p(\tau) \\
& \frac{x_{\alpha}}{r_{\alpha}^{2}} \epsilon^{\prime \prime}+\alpha^{\prime \prime}+2 \zeta_{\alpha} \frac{1}{U} \alpha^{\prime}+\frac{1}{U^{2}} M(\alpha)=r(\tau)
\end{aligned}
$$

Here, $\alpha$ and $\epsilon$ are non-dimensional pitch and plunge, $U$ is the non-dimensional flow speed and $\tau$ is non-dimensional time. $x_{\alpha}$ is the distance from the elastic axis to centre of mass of an airfoil, $r_{\alpha}^{2}$ is the radius of gyration, $\bar{\omega}$ is ratio of plunge to pitch natural frequencies. $\zeta_{\epsilon}$ and $\zeta_{\alpha}$ are the viscous damping ratios in plunge and pitch, $p(\tau)$ and $r(\tau)$ are aerodynamic coefficients can be found in [8] and are not repeated here. $M(\alpha)$ in Eq. (2) is replaced by a bilinear function to introduce freeplay structural nonlinearity $[2,3]$. The restoring moment as a function of pitch is given by:

$$
M(\alpha)=\left\{\begin{array}{ccc}
\alpha+\delta, & \text { if } & \alpha<-\delta \\
0, & \text { if } & |\alpha| \leq \delta \\
\alpha-\delta, & \text { if } & \alpha>\delta
\end{array}\right.
$$


Here $M(\alpha)$ is the restoring moment due to torsional spring, $\alpha$ is nondimensional pitch angle and $\delta$ is freeplay length. The freeplay length $\delta=.0087$ is fixed for all our numerical simulations.

\section{Stability analysis under deterministic flow}

\subsection{Nonlinear bifurcation analysis}

The numerical bifurcation analysis is done for bilinear structural nonlinearity under deterministic free stream velocity. As predicted earlier by [3], the system exhibits wide variety of dynamical phenomenon well below linear flutter speed. Indeed, when $U<U_{c r}$ a plethora of dynamical responses are observed. For example, at $U=3$ the airfoil exhibits chaotic motion as shown in Figs. 1 (a) and (b). Increasing $U$ to 4, a period doubling cascade is observed (see Figs. 1 (c) and (d)). Further increasing the flow speed such that $U=5$, sustained LCOs are encountered, and the corresponding phase plots and time histories are shown in Figs. 1 (e) and (f). It is worthwhile to note that only representative time histories and phase plots are shown in Fig 1 for the sake of brevity. A finer resolution of the input flow speed results in another set of distinct dynamics that shows dependency on the initial conditions. However, the present study focuses only on the broad dynamics that are possible in a base-line, freeplay aeroelastic system for comparison against the stochastic dynamics. The authors recommend the interested readers to refer $[3,8]$ for excellent discussions on bifurcation scenarios in aeroelastic systems with freeplay nonlinearity.

\subsection{Stochastic response analysis}

So far an investigation into the bifurcation scenarios in the deterministic system was carried out.

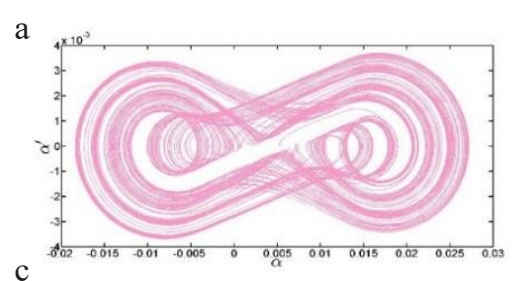

C

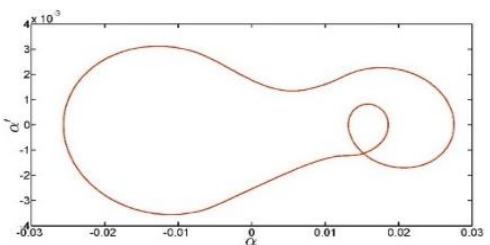

e

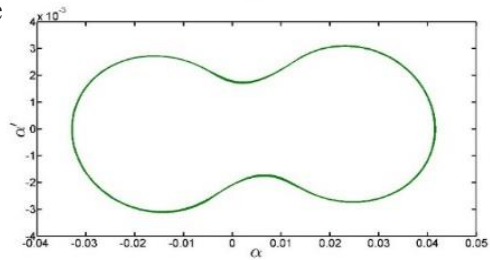

b

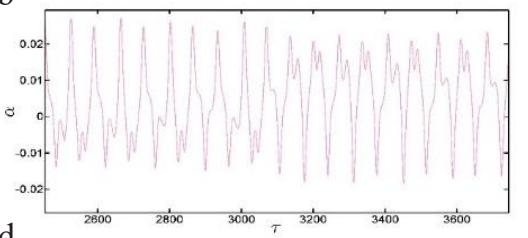

d

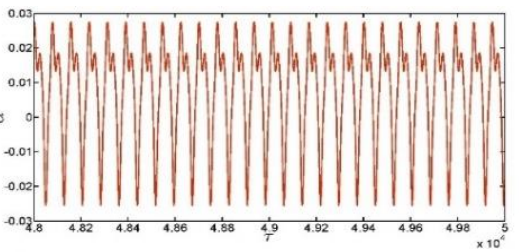

$\mathrm{f}$

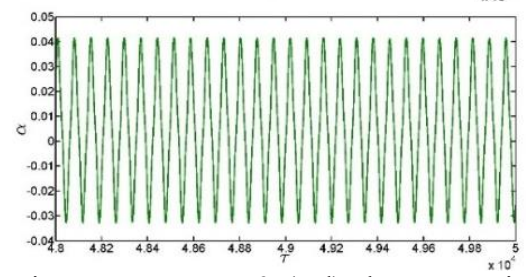

Fig. 1. (a,b) Phase portrait and time response of chaotic response at $U=3$, (c,d) phase portrait and time response period doubling at $U=4$, and (e,f) phase portrait and time response limit cycle oscillation at $U=5$. 
Next, the input flow is considered to be a randomly time varying parameter modelled as a long time scale fluctuation. The details of the fluctuation model can be found in [7]. Once again the time responses are obtained by using a fourth order Runge Kutta algorithm. With mean flow speed as the bifurcation parameter, sample cases of time histories are shown in Fig 2.

a

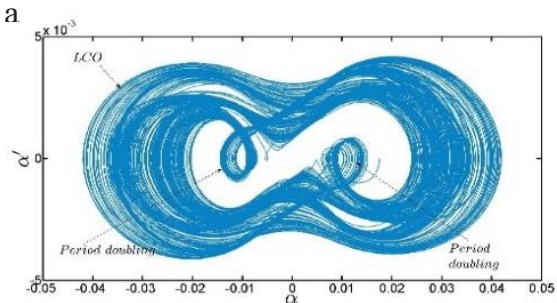

C $5 \times 10^{2}$
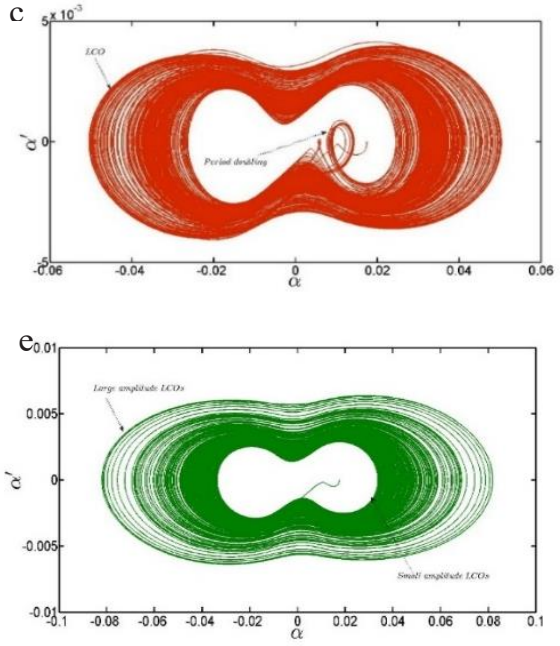

b
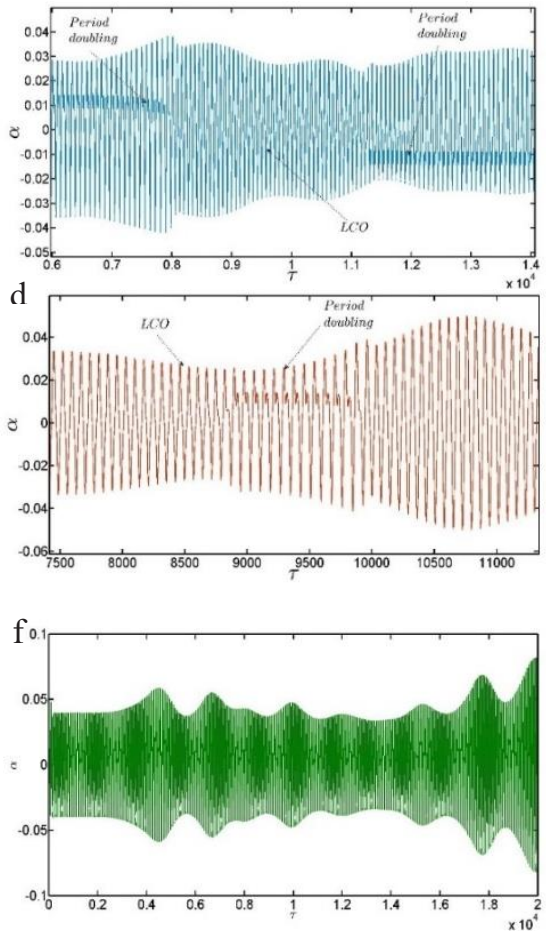

Fig. 2. Phase plots and time responses for $U_{m}=4.4(\mathrm{a}, \mathrm{b}), 4.97(\mathrm{c}, \mathrm{d})$ and $5(\mathrm{e}, \mathrm{f})$.

At $U_{m}=4.4$, the response shows a switching between a period doubling to LCO and to another period doubling attractor in a random fashion (see Fig. 2a). Such an alternation between qualitatively distinct dynamics can be attributed to the presence of randomly fluctuating bifurcation parameter (here the flow speed). Indeed, the flow speed is time varying with scales of fluctuation that are much larger than the system time scale. This permits the flow speed to go back and forth into different attractor regimes in an unpredictable fashion. The ability of long time scale flow fluctuations to manifest distinct switching between attractors have been investigated by Venkatramani et al. [7]. It is worth pointing out that such stochastic responses are a signature of a noise induced phenomenon called intermittency. A typical noise induced intermittency is known to possess "on" and "off" states - characterized in terms of sudden periodic oscillations amidst near rest states. Such responses have been reported in the literature [8,7]. However, an underlying requirement for "on-off" type intermittency is the presence of supercritical Hopf bifurcation in the baseline, deterministic aeroelastic system (as in the presence of cubic hardening nonlinearity). Given the non-smooth nature of nonlinearity considered in the present study, wherein, the deterministic system possesses multiple attractors, it is unsurprising that a switching between two or more attractor regimes is observed. A rudimentary observation would indicate that the nature and strength of the noise would exacerbate the extent of switch 
over between these attractor spaces. However, it would be premature to comment on the same without carrying out a systematic investigation.

Increasing the mean flow speed further to 4.97 , it is observed that a switching between qualitatively distinct regimes still persists (see Fig. 2b). However, the underlying attractor regimes are distinct from those observed in Fig. 2a. Here, an alternation of the dynamics between an LCO attractor and period doubling exist. Note that this response is an intermittent switching as well. Next, $U_{m}=5$ one can observe a large amplitude periodic response with varying amplitudes (see Fig. 2c). In other words, such responses possess LCOs that are of relatively higher amplitudes interspersed amidst a lower amplitude LCO. From the parlance of stochastic dynamical systems, these responses are a signature of a random LCO [5]. From an engineering perspective, these responses indicate the onset of oscillatory instabilities in the stochastic aeroelastic system. Once the stochastic responses are obtained it becomes imperative that any comments on the bifurcation characteristics would involve a stochastic bifurcation analysis. As a first step, we carry out a Phenomenological or P-type bifurcation analysis. This involves tracking the topological changes in the structure of the joint probability density function (j-pdf) of the state variables as the bifurcation parameter is varied. For a clearer visualization, the two dimensional section of the $\mathrm{j}$-pdfs are shown in Fig.6 in terms of the contour plots. The contour plots correspond to those time histories shown in Fig. 5. It is pertinent to note that a visual inspection of these contour plots clearly reveal a topological change in their structure. Such a change is an indication of a P-bifurcation occurring in the aeroelastic system with freeplay nonlinearity excited with stochastic wind.
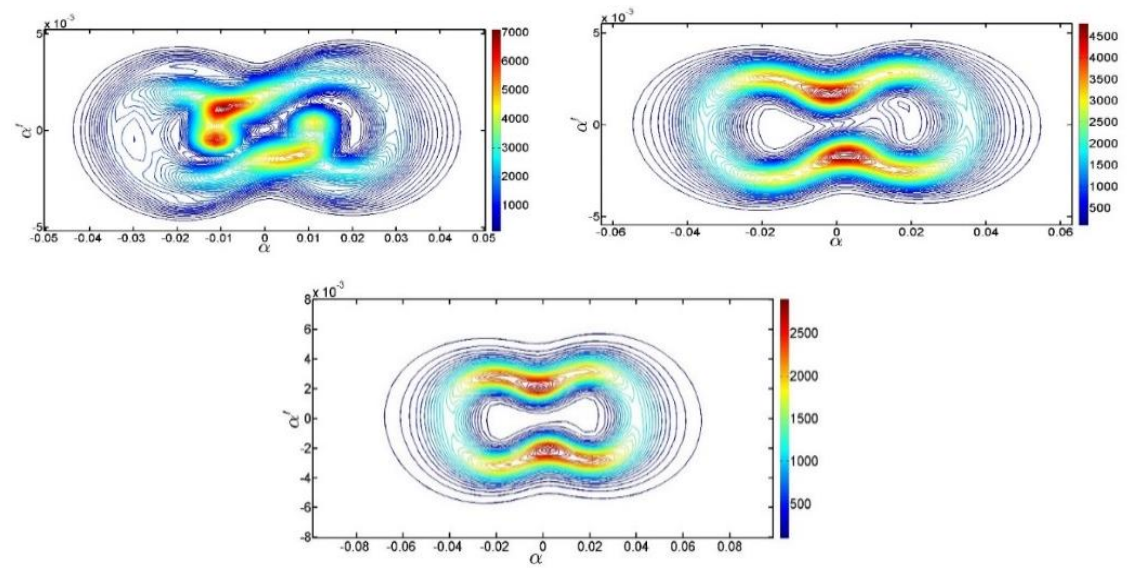

Fig. 3. Contour plots corresponding to Fig. 2.

\section{Concluding remarks}

A pitch-plunge airfoil with free Play nonlinearity in the pitch is investigated in the presence of randomly fluctuating wind. The deterministic bifurcation analysis revealed that the preflutter response possess a wide spectrum of nonlinear responses such as chaos, period doubling and LCOs. The introduction of fluctuating flows were observed to give rise to intermittent switching between LCOs and period doubling in a random fashion. Such responses were a signature for intermittency and were observed to presage the random LCOs. The stochastic responses were further discussed in terms of P-bifurcation concepts. It would be interesting to investigate the stochastic bifurcations in terms of quantitative measures such as Shannon entropy and Largest Lyapunov exponent. These investigations can provide deeper insights and are to be taken up in future. 


\section{References}

[1] Y.C. Fung, An introduction to the theory of aeroelasticity, 1955. Dover (1969)

[2] R. Vasconcellos, A. Abdelkefi, M.R. Hajj, F.D. Marques, CNSNS, 19, 5 (2014)

[3] H. Alighanbari, S.J. Price, Nonlinear Dyn, 10, 4 (1996)

[4] S.J. Price, H. Alighanbari, B.H.K. Lee, J Fluids Struct, 9, 2 (1995)

[5] D. Poirel, S.J. Price, AIAA J., J Aircr, 34, 5(1997)

[6] J. Venkatramani, Sunetra Sarkar, Sayan Gupta, Nonlinear Dyn, 1-17 (2018)

[7] J. Venkatramani, J.S. Krishna Kumar, Sunetra Sarkar, J Fluids Struct, 75, 9-26 (2017)

[8] H. Alighanbari, PhD thesis, Mc Gill University, Montreal (2001) 